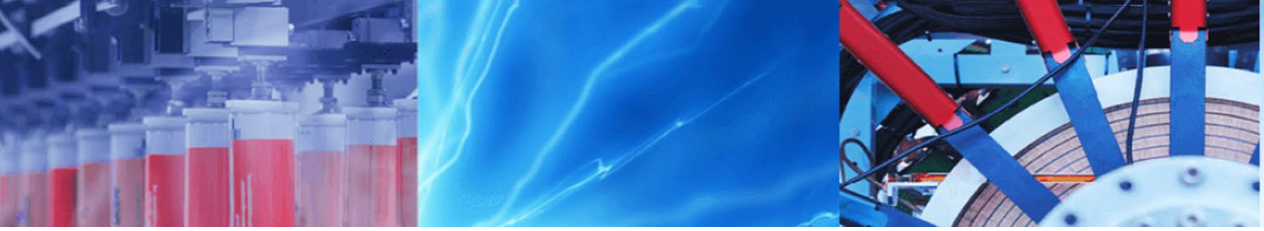

Editorial

\title{
Editorial of the topical collection "state of the art on autonomous robot systems and competitions"
}

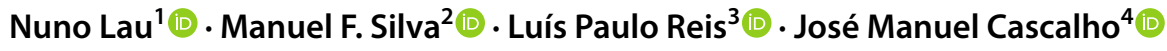

Accepted: 22 September 2021

Published online: 01 October 2021

(c) The Author(s) $2021 \quad$ OPEN

In the last couple of decades, autonomous robots have seen a significant increase in its use in the most diverse fields of application, but several problems remain open. This has stimulated the academic community to invest in areas related with robotics and its support technologies, and competitions have been organized around the world as a means of advancing the state of the art on these topics. Given this scientific environment, journals and conferences broadly disseminate the developed knowledge.

Organized since 2001, in parallel with the Portuguese Robotics Open, the IEEE International Conference on Autonomous Robot Systems and Competitions (ICARSC) gathers participants from academia and industry working in the field of autonomous robot systems and related areas. It aims at exploring and discussing the latest trends in research and technology in the fields of robotics and related areas.

The authors of the best papers presented at ICARSC'2020 were invited to submit extended and improved versions of their manuscripts to this Topical Collection (TC) of the SN Applied Sciences.

The eight papers included in this TC cover distinct application topics, ranging from the proposal of a modular framework to generate robust biped locomotion, considering obstacles to plan all the walking reference trajectories, to the computational complexity analysis of two recently proposed heuristics (based on the Potential Grasp Robustness (PGR) metric) for underactuated hands grasp pose selection, to the generation of a more realistic dataset for learning setplays from demonstration for the BahiaRT robotic soccer team, including the definition of better pass and defensive strategies. Other areas are also addressed, such as methods for real-time door state classification able to differentiate between open, closed, and semi-open doors, that improve robot navigation in indoor spaces, working in low-powered computers, for producing multi-domain maps for the inspection of offshore wind farms, using an Autonomous Surface Vehicle (ASV) and fusing the information from navigational sensors to create a more complete and more reliable map that maximizes the observability of all the offshore structures from the wind turbines to the cable arrays, designing a multi-agent system to promote regular physical activity habits among the elderly, which not only persuades the users to perform exercise routines, but also guides and accompanies them during exercises to provide effective training and engagement to the user, and also introducing a realistic micromouse simulator, having hardwarein-the-loop capabilities, which aims to be simple to use, customizable, and designed to be as similar as possible to the real robot configuration. Finally, the development of an autonomous wheelchair for patient's transportation on healthcare institutions is described, which can receive transportation requests directly from the hospital information management system, pick up patients at their beds, navigate autonomously through different floors, avoid obstacles, communicate with elevators, and drop patients off at the designated operating room.

Summing up, this TC presents peer reviewed contributions on the state of the art, from theory, to prototyping,

$\triangle$ Manuel F. Silva, mss@isep.ipp.pt| ${ }^{1}$ University of Aveiro, Aveiro, Portugal. ${ }^{2}$ School of Engineering of the Porto Polytechnic, Porto, Portugal. ${ }^{3}$ University of Porto, Porto, Portugal. ${ }^{4}$ University of the Azores, Ponta Delgada, Portugal.

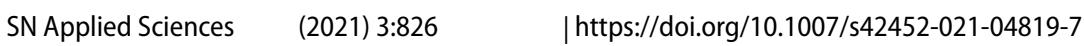


to final product development, that were presented at the ICARSC'2020 - The 20th IEEE International Conference on Autonomous Robot Systems and Competitions.

The guest editors would like to thank all paper reviewers for their efforts in reviewing the author's submissions, and all authors of the invited papers for their positive response to the forward comments and suggestions. Also, special thanks are due to Thomas von Larcher for his support to this TC, which it is hoped will constitute a valuable addition to scientific and developmental knowledge in autonomous robot systems and competitions.

\section{Declarations}

Conflict of interest The authors declare that they have no Conflict of interest.
Open Access This article is licensed under a Creative Commons Attribution 4.0 International License, which permits use, sharing, adaptation, distribution and reproduction in any medium or format, as long as you give appropriate credit to the original author(s) and the source, provide a link to the Creative Commons licence, and indicate if changes were made. The images or other third party material in this article are included in the article's Creative Commons licence, unless indicated otherwise in a credit line to the material. If material is not included in the article's Creative Commons licence and your intended use is not permitted by statutory regulation or exceeds the permitted use, you will need to obtain permission directly from the copyright holder. To view a copy of this licence, visit http://creativecommons. org/licenses/by/4.0/.

Publisher's Note Springer Nature remains neutral with regard to jurisdictional claims in published maps and institutional affiliations. 